\title{
Design of intelligent vehicle control system based on machine visual
}

\author{
Ai-Juan $\mathrm{Li}^{1,2, a}$, Wan-Zhong Zhao ${ }^{2, b, \dagger}$, Si-Ming Fang ${ }^{3, c}$, Gang Xu ${ }^{1, d}$ \\ and Hui-Jun Wang ${ }^{1, e}$ \\ ${ }^{1}$ School of Automotive Engineering, Shan Dong Jiaotong University, \\ Jinan 250023, China \\ ${ }^{2}$ Energy and Power Engineering College, Nanjing University of Aeronautics \& \\ Astronautics, Nanjing 210016, China \\ ${ }^{3}$ Hao Tai Investment Group co., LTD, Kelamayi 834000, China \\ aliaijuan2008@163.com, ${ }^{b}$ zhaowanzhong@126.com, ${ }^{c h t f s m @ 163 . c o m, ~}{ }^{d}$ \\ zyjnjds@163.com, ${ }^{e}$ wanghj@sdjtu.edu.cn
}

\begin{abstract}
In order to improve the control system's accuracy and adaptability of the intelligent vehicle, this paper designs the control system of the smart intelligent car. In this paper, a control system of the smart intelligent car is designed, the control system including hardware system and software system. The design of the hardware system design includes the design of power supply module, data acquisition module and motor control module design. The design of software system includes the main program design, image acquisition procedures and speed control program design. The control system is applied to the smart intelligent car to test the control system's feasibility. The test results showed that compared with the car without using the control system designed in this paper, the intelligent smart vehicle can accurately move along the centerline of the circuit.
\end{abstract}

Keywords: Intelligent vehicle; Control system; Machine visual; Design.

\section{Introduction}

In recent years, the intelligent vehicle technology becomes improve and perfect constantly, intelligent vehicle plays a more and more important role in industrial production and daily life $[1,2]$. Control system is one of the key technologies of intelligent vehicle, it mainly to control the vehicle to track the reference trajectory [3, 4]. Machine vision belongs to the perceptual system of intelligent vehicle, mainly to carry out on the driving environment of vehicle and transmitted the environmental information to the control system, control system control the vehicle to drive according to the environmental information [5-7]. In this paper, the intelligent vehicle's control system is designed based on machine vision. The CCD cameras can obtain the image information effectively and then transfer the image information to the control system, the control system makes decision to control the car to trace. The system can realize the tracking control 
of the intelligent vehicle, intelligent vehicle control become more accurate and efficient.

\section{The Design of the Intelligent Vehicle Control System}

In this paper, the intelligent vehicle control system based on Freecale32 bit MCU K60, the image acquisition module get image information based on CCD camera and track in PD feedback control of steering gear steering motor speed control by using PID control through the PWM control power of the motor drive circuit and through the analysis of the algorithm software specific out the road ahead to aid the debugging system for the optimized software algorithm, make a decision of the optimal moving path for the car finally. Fig. 1 is the system frame of the intelligent vehicle.

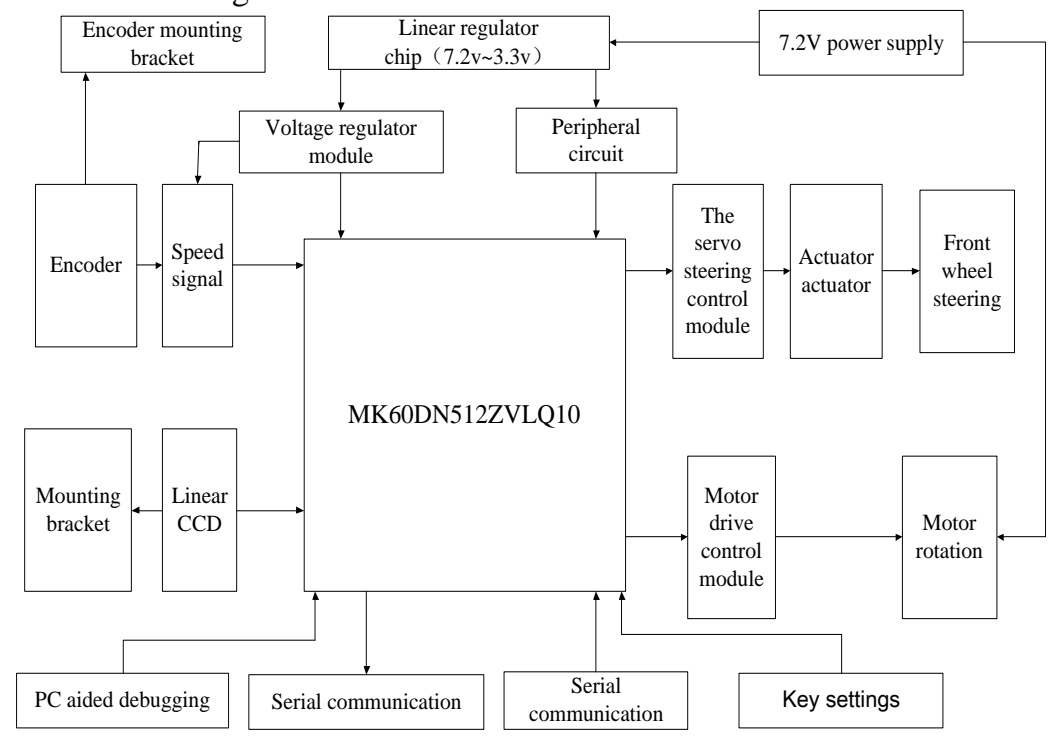

Fig. 1. System frame diagram of intelligent vehicle

\section{Intelligent Vehicle Control System's Design}

Intelligent vehicle control system's design includes hardware design and software design. In order to play a good performance of the smart intelligent car, the control system's requirement is: efficient, accurate, stable and simple.

\section{Hardware Design}

The smart car's control system contains a linear CCD camera, SD-5 server and 540 motors and other major components. In order to make it better to coordinate 
with each other and give full play to the performance of the components. Hardware system is designed on the basis of the mk60 main control chip, power supply circuit, linear CCD image acquisition, motor drive, speed detection, servo control, serial communications and external LCD display, key input module. Fig. 2 is a whole hardware system for intelligent vehicles.

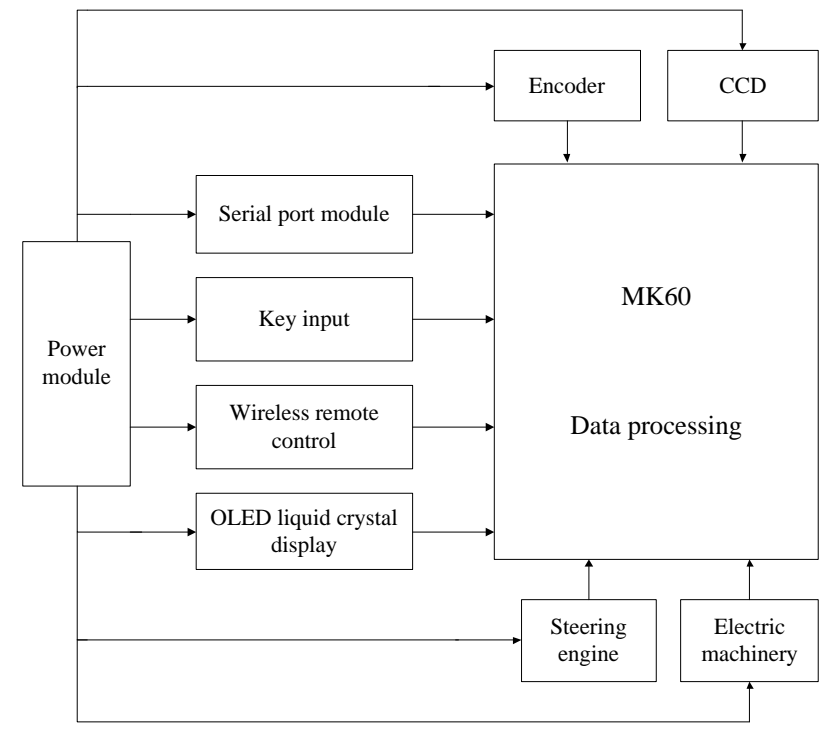

Fig. 2. Overall hardware system for intelligent vehicle

\subsection{Power module}

Linear CCD, encoder, etc. are used for auxiliary sensor data acquisition, wireless serial communication, wireless remote control and keys input debugging module of voltage requirements is not so high, the smart car on the use of the $\operatorname{lm} 2940$ regulator chip, to provide the required voltage.

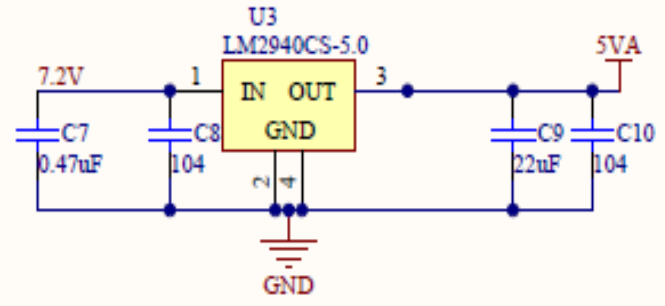

Fig. 3. LM2940-5V circuit diagram 


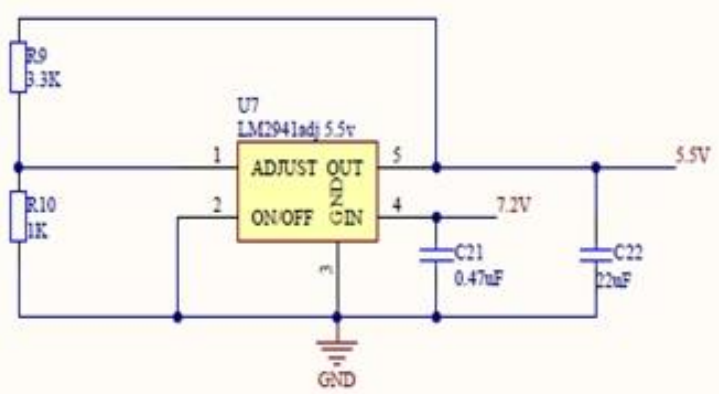

Fig. 4. Servo regulator circuit diagram

Due to the special requirements of SD-5 steering gear, power supply voltage shall not be more than $5.5 \mathrm{~V}$. In order to improve its response speed, the smart car adopts LM2941 chip, build $5 \mathrm{~V}$ voltage regulator circuit to meet the needs of the steering gear. Figure 3 is the LM2940-5V circuit diagram and figure 4 is the servo regulator circuit diagram.

Motor power demand is bigger, directly use $7.2 \mathrm{~V}$ battery power, large current and voltage stability can meet the normal requirements of the electrical machine.

\subsection{Data acquisition module}

The smart car uses the linear CCD camera in the track information to identify the path. Its principle is that the CCD camera acquires the image every $10 \mathrm{~ms}$ on the track, the camera gets the traffic information in front of the car and transmits to MCU. According to the type of collected track for processing and implement corresponding control algorithm, the MCU make the car run quickly and efficiently. Fig. 5 is CCD module op-amp circuit diagram.

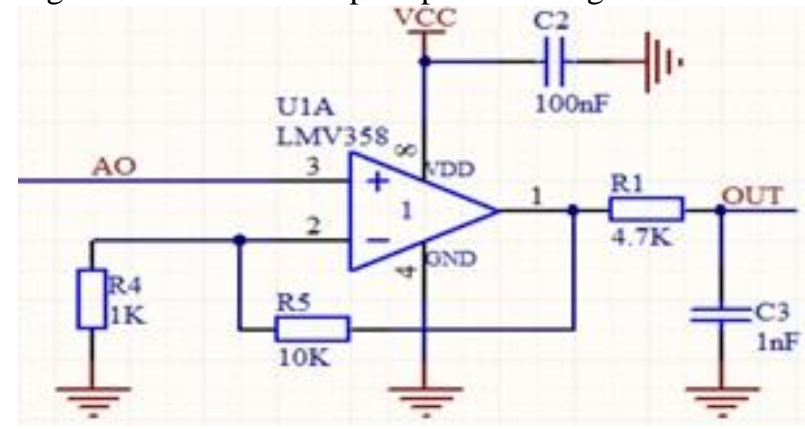

Fig. 5. CCD module op-amp circuit diagram

\subsection{Motor control module}

Motor drive circuit for the speed control of intelligent vehicle have a vital role, which has better braking and accelerating ability with great help to improve the 
speed of the car. The speed of the motor is proportional to the voltage on the motor, the output torque is proportional to the current of motor. Because in the process of the intelligent car driving to change the rotating speed of DC motor, adopting a PWM (pulse width modulation) square wave, on the direct current (DC) on the duty ratio of PWM wave replied needed for the intelligent car speed, the motor have the effect of a low pass filter, the PWM signal is converted to DC level effectively. PWM signal can be produced by K60 microcontroller, with accurate pulse width can adjust to the rotation speed of dc motor, and to optimize the frequency of the PWM signal, in order to prevent the motor shaking. The current direction of DC motor is replaced can control the rotation direction of the DC motor.

\section{The Software Design}

The control system software is mainly to complete the car image information acquisition and the speed control. In order to make the image process, the normalization of data is used, the line is made clearer. In order to control the vehicle, the classic PID control algorithm is used. The result of the theory and practice is compared, and appropriate compensation is added, the smart car is controlled to track the path to achieve the effect of steady and rapid. Fig. 6 for the owner program control flow chart.

\subsection{Track center line image extraction}

In the course of the intelligent car, the track information is acquired through the CCD camera, at the same time, there will be some interference or information that is not available. This needs to filter out the information of the track before extracting the track information, and then we can effectively identify the track, so we have to deal with the track. Fig. 7 is the circuit center line extraction flow chart.

\subsection{Speed control}

Speed control and image acquisition are inextricably linked, microcontroller according to judge the type of image to choose different control algorithms to optimize the control of the motor. Fig. 8 is a flowchart of the speed control. 


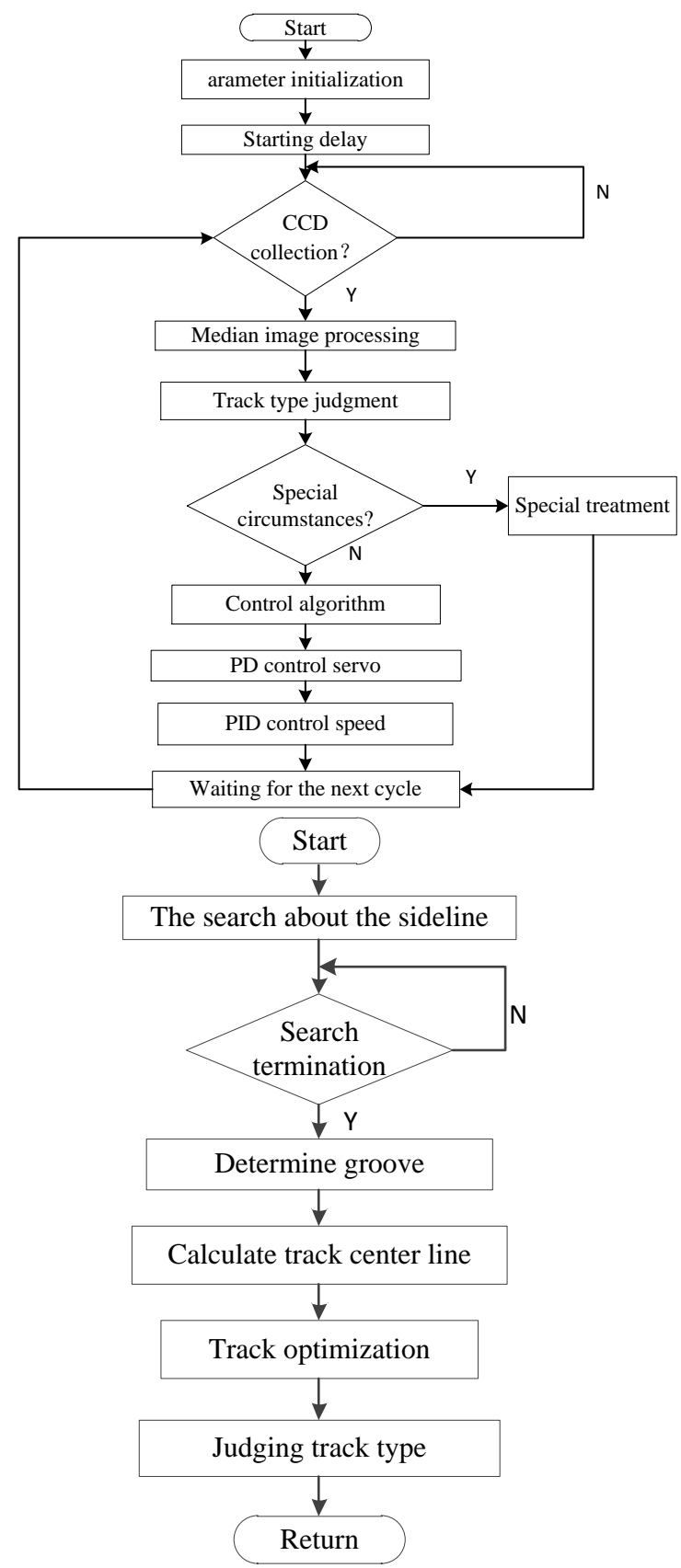

Fig. 6. Vehicle master program control flow chart
Fig. 7. Circuit center line extraction flow chart 


\section{Experimental Verification and Analysis}

The test of the intelligent vehicle used and not used the designed control system on the standard circuit respectively is shown in this paper. The speed of the intelligent vehicle is $2.5 \mathrm{~m} / \mathrm{s}$, the test result of two car track diagram is shown in Fig. 9. The test results showed that compared with no designed intelligent car, the performance of stability, accuracy, sensitivity and adaptability of the designed intelligent vehicle can have significantly improved.

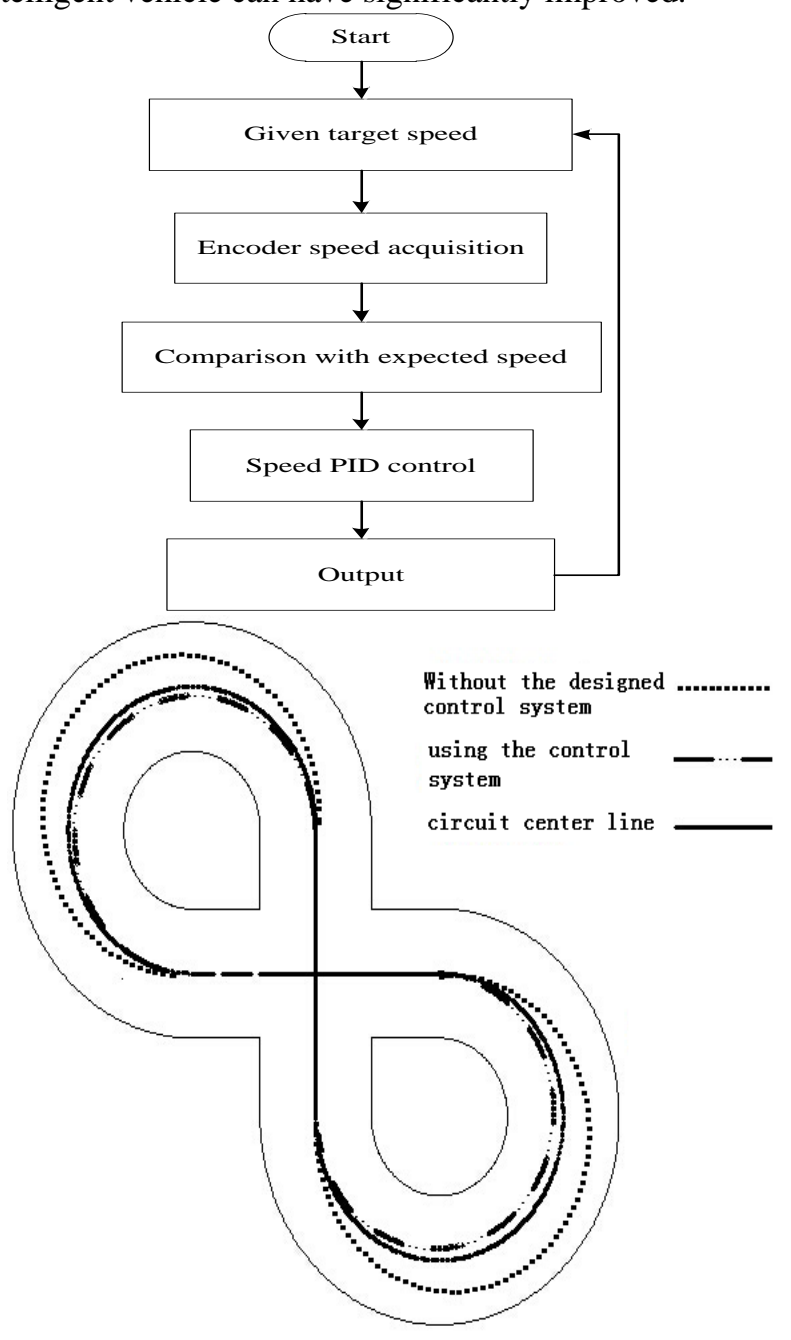

Fig. 8. Flowchart of the speed control

Fig. 9. Comparison Chart of the experimental trajectory 


\section{Conclusions}

In this paper, the intelligent vehicle control system is tested in the standard track, we can get the conclusion just as follows:

(1) This paper has designed the intelligent vehicle control system's the hardware and software. The designed control system can realize the automatic control of the intelligent vehicle to meet the automatic control requirement of the intelligent vehicle.

(2) The experimental results showed that compared with the intelligent car without the designed control system, this paper's control system can make the intelligent vehicle drive along the circuit stably and accurately under high speed.

\section{Acknowledgments}

This project is supported by National Natural Science Foundation of China (Grant No. 51375007 and 51505258), Science and Technology Development Projects of Shandong Province, China (Grant No. 2012G0020504), Research Project of State Key Laboratory of Mechanical System and Vibration (Grant No. MSV201507), Key Research and Development Project of Shandong Province, China (Grant No. 2015GGX105010), The Ministry of Transport Applied Basic Research Project, People's Republic of China (Grant No. 2013319817190).

\section{References}

[1]. Ren D. B., Zhang J. Y., Zhang J. M., et al. Trajectory planning and yaw rate tracking control for lane changing of intelligent vehicle on curved road, Science China (Technological Sciences). 54 (2011) 630-642. (In Chinese)

[2]. ShenH. ,LingR.,LiS.M..Steering Control on Large Curvature Road Based on Preview Optimal Curvature Model, China Mechanical Engineering. 23 (2012) 2111-2115, 2116. (In Chinese)

[3]. GUO Lei, LI Keqiang, WANG Jian qiang, et al. Lane Detcction Method by Using Steerable Filter, Chinese Journal of Mechanical Engineering, 44 (2008) 214-218, 226. (In Chinese)

[4]. Broggi A, Cattani S.An agent based evolutionary approach to path detection for off-road vehicle guidance, Pattem Recognition Letters. 27 (2006) 1164-1173.

[5]. Ai Ning, Qu Shaocheng, Liu Dong, et al. Research on the path identification and tracking of intelligent vehicle based on CCD camera, electronic measurement technology, 32 (2009) 77-80. (In Chinese)

[6]. Bao FA sun, Zhang Xiaoling, etc... With the hardware system design of the camera tracking of the intelligent vehicle, Value engineering.31 (2012) 
201-202. (In Chinese)

[7]. Hu M K. Visual pattern recognition by Moment invariants, IRE Transactions on Information Theory. 8(1962) 179-187. 